\title{
ON THE EXISTENCE OF TRANSIENT SOLUTIONS OF A TUNNEL FIRE MODEL*
}

\author{
INGENUIN GASSER ${ }^{\dagger}$ AND HERBERT STEINRÜCK ${ }^{\ddagger}$
}

\begin{abstract}
In this paper we present a global existence and uniqueness result of solutions to a system consisting of a coupled nonlinear PDE and a nonlinear ODE. This system is a one-dimensional fluiddynamic model for tunnel fires and was presented in [6] and then anaylsed in [2] and [5] with respect to the applications.
\end{abstract}

Key words. Gasdynamics, Low-Mach-number, Fire in Tunnels

Subject classifications. 76D05, 76N15; 35Q30, 80A20.

\section{Introduction}

Due to some serious fire accidents in the near past tunnel fires have become an interesting topic not only for CFD engineers. In the last decades various mathematical models based on a gas-dynamic description of the air in the tunnel have been proposed. A good overview on the modeling approaches is given in $[4,8]$. A recent survey on computer codes for tunnel fire simulations can be found in [7]. In this context the internet platform [1] should be mentioned also.

On one hand complex CFD codes need detailed (often non-available) input data and produce very detailed flow results for a single situation. Nevertheless, many questions from the application cannot be answered. On the other hand such codes are expensive and need a lot of know how to be used. These are some of the reasons why there is a revival of simple one-dimensional fluid dynamic models which are fast and in general not worse than CFD codes in answering certain questions from the application.

One of these one-dimensional models was introduced in [6]. To our knowledge this is one of the first models derived from underlying fluid dynamic equations. The single dimension refers to the longitudinal spacial extension. This model is derived in such a way that it allows us to combine both a good description in the low Mach number regime and significant heat transport. The results obtained by now show that the model seems to keep the main features of tunnel fires. A good (at least qualitative) agreement with results from experiments has been obtained (see [6, 3]). Stationary solutions of the model equation have been discussed in [2].

Let $\rho, u, p, T$ be the (dimensionless) density, velocity, pressure and the temperature of the flow in the tunnel, respectively. Then the leading order equations in a small Mach number expansion are given by

$$
\begin{aligned}
\rho_{t}+u \rho_{x} & =-\rho q, \\
u_{t}+u u_{x}+\frac{1}{\rho} p_{x} & =-p_{d v} \frac{u|u|}{2}-f_{d} \sin \alpha \\
u_{x} & =q
\end{aligned}
$$

*Received: April 26, 2006; accepted (in revised version): July 22, 2006. Communicated by Shi Jin.

${ }^{\dagger}$ Department Mathematik, Universität Hamburg, Bundesstraße 55, 20146 Hamburg, Germany (gasser@math.uni-hamburg.de).

${ }_{\ddagger}^{\ddagger}$ Institut für Strömungslehre und Wärmeübertragung, Technische Universität Wien, Resselgasse 3, A-1040 Wien, Austria (herbert.steinrueck@tuwien.ac.at). 
with $q=q(x, t)$ as time and space dependent (scaled) heat source. The equations have to be supplemented by the equation of state for an ideal gas $p=\rho T$. In the equation of motion we have considered the pressure loss $p_{d v} u|u| / 2$ and the buoyancy force $f_{d} \sin \alpha$ with a scaled pressure loss coefficient $p_{d v}$ and a (scaled) buoyancy parameter $f_{d}$. For more details on the derivation of the model and scaling see $[6,3]$.

As far as boundary data is concerned we prescribe Dirichlet data for the pressure $p$ at the entrance and the exit, homogenous Neumann conditions for the velocity $u$ (no fire at the entrance and exit) and standard inflow boundary conditions for the density $\rho$ (for $t>0)$

$$
\begin{gathered}
p(t, 0)=p_{l}(t), p(t, 1)=p_{r}(t), \\
u_{x}(t, 0)=0, u_{x}(t, 1)=0, \\
\rho(t, 0)=\rho_{r}(t) \text { if } u(t, 0)>0, \rho(t, 1)=\rho_{r}(t) \text { if } u(t, 1)<0 .
\end{gathered}
$$

Initial data are prescribed for the density and the velocity

$$
u(0, x)=u_{0}(x), \quad \rho(0, x)=\rho_{0}(x), \quad \forall x \in[0,1] .
$$

Thus, our model consists of the equations (1.1)-(1.3), the boundary conditions (1.4)(1.6) and the initial conditions (1.7).

A first problem lies in the fact that we have two boundary conditions for the pressure but only a first derivative of the pressure in the model. To get rid of this problem we consider the following reformulation (restricted to one space dimension). We eliminate the pressure by multiplying the equation (1.2) by $\rho$ and integrating over $x \in[0,1]$. This gives

$$
\int_{0}^{1} \rho u_{t} d x+\int_{0}^{1} \rho u u_{x} d x+p_{r}-p_{l}=-\int_{0}^{1} p_{d v} \rho \frac{u|u|}{2} d x-\int_{0}^{1} f_{d} \sin \alpha \rho d x .
$$

Equation (1.2) gives

$$
u(x, t)=v(t)+\int_{0}^{x} q(y, t) d y=v(t)+Q(x, t)
$$

where $Q(x, t)$ is a known function. Then we obtain the system

$$
\begin{aligned}
\rho_{t}+(v+Q) \rho_{x} & =-\rho q \\
R v_{t}+R_{q} v+\int_{0}^{1} p_{d v} \rho \frac{(v+Q)|v+Q|}{2} d x & =-R_{Q_{t}+Q q+f_{d} \sin \alpha}-p_{r}+p_{l}
\end{aligned}
$$

for $\rho$ and $v$ where $R, R_{q}, R_{Q_{t}+Q q+f_{d} \sin \alpha}$ denote functionals applied to $\rho(x, t)$ defined in (2.1)- (2.2). This system consists of an Ode for $v$ and a Pde for the density $\rho$. The only boundary conditions needed are the inflow conditions (1.6) for the continuity equation. The conditions on the pressure appear as parameters in (1.11). The condition on the velocity (1.5) is automatically fulfilled by (1.9). In this paper we use this last formulation (1.10)-(1.11) for the analysis.

The paper is organized as follows. In section 2 we present the existence and uniqueness result.

\section{Global existence and uniqueness}

In this section we focus on the global existence and uniqueness of solutions of the equations (1.1)-(1.2) with boundary conditions (1.4)-(1.6) and initial conditions (1.7).

We formulate the following conditions: 
(A1) On the data in the equations:

(i) Let $q=q(x, t) \geq 0$ be a smooth function on $[0,1] \times[0, \infty)$ with $\operatorname{supp} q(., t) \subset$ $(0,1) \forall t \in[0, \infty)$.

(ii) Let $p_{d v}=p_{d v}(x)$ be a smooth function on $[0,1]$ with $p_{d v}(x)>0 \quad \forall x \in$ $[0,1]$.

(iii) Let $\alpha=\alpha(x)$ be a smooth function on $[0,1]$ with $-\frac{\pi}{2}<\alpha(x)<\frac{\pi}{2} \quad \forall x \in$ $[0,1]$.

(A2) On the boundary data:

(i) Let $\rho_{l}=\rho_{l}(t)>0$ and $\rho_{r}=\rho_{r}(t)>0$ be in $C^{1}[0, T]$ for all $T>0$.

(ii) Let $p_{l}=p_{l}(t)$ and $p_{r}=p_{r}(t)$ be smooth functions for $t \in[0, \infty]$.

(A3) On the initial data:

(i) Let $u_{0}=u_{0}(x)=v_{0}+\int_{0}^{x} q(y, 0) d y$ for some constant $v_{0}$.

(ii) Let $\rho_{0}=\rho_{0}(x)>0$ be a piecewise continuous differentiable function for $x \in[0,1]$.

In the following we use the notation

$$
\begin{aligned}
R(t) & =\int_{0}^{1} \rho(x, t) d x \\
R_{f}(t) & =\int_{0}^{1} \rho(x, t) f(x, t) d x .
\end{aligned}
$$

Before presenting the main existence result we state a result concerning lower and upper bounds of the solutions of the continuity equation (1.10). In the following we use the notation $f_{\max }(T)=\max _{(x, t) \in[0,1] \times[0, T]} f(x, t)$.

Lemma 2.1. Let (A1)(i) hold. Let $T>0$. Then for a given $v \in C[0, T]$ the continuity equation (1.10) with initial $\rho_{0}$ and boundary data (1.6) has a unique piecewise continuously differentiable solution $\rho$ on $(x, t) \in[0,1] \times[0, T]$. It satisfies

$$
0<\rho_{\min }(T) \leq \rho(x, t) \leq \rho_{\max }(T), \quad \forall(x, t) \in[0,1] \times[0, T],
$$

with

$$
\begin{aligned}
& \rho_{\min }(T)=\min _{t \in[0, T], x \in[0,1]}\left(\rho_{l}(t), \rho_{r}(t), \rho_{0}(x)\right) e^{-q_{\max } T}, \\
& \rho_{\max }(T)=\max _{t \in[0, T], x \in[0,1]}\left(\rho_{l}(t), \rho_{r}(t), \rho_{0}(x)\right) .
\end{aligned}
$$

We also bring the proof since it involves the characteristics where the main ideas of the existence analysis are based on.

Proof. Consider a time interval $[0, T]$. The characteristic curves $\eta=\eta\left(t, x_{0}, t_{0}\right)$ are defined by

$$
\frac{d \eta\left(t, x_{0}, t_{0}\right)}{d t}=u\left(\eta\left(t, x_{0}, t_{0}\right), t\right)=v(t)+Q\left(\eta\left(t, x_{0}, t_{0}\right), t\right), \quad\left\{\begin{array}{l}
\eta\left(0, x_{0}, 0\right)=x_{0}, \\
\eta\left(t_{0}, 0, t_{0}\right)=0, \\
\eta\left(t_{0}, 1, t_{0}\right)=1 .
\end{array}\right.
$$

Given a continuous $v=v(t)$ the right hand side in (2.6) is continuous (in $\eta$ and $t$ ) and Lipschitz-continuous in $\eta$. The Lipschitz constant $L$ satisfies $L \leq q_{\max }(T)$. Therefore the characteristic curves are uniquely defined at every point $(x, t)$ and do not intersect. 
The continuity equation (1.10) reads along the characteristics

$$
\frac{d \rho\left(\eta\left(t, x_{0}, t_{0}\right), t\right)}{d t}=-q\left(\eta\left(t, x_{0}, t_{0}\right), t\right) \rho\left(\eta\left(t, x_{0}, t_{0}\right), t\right)
$$

with

$$
\begin{aligned}
& \rho\left(\eta\left(0, x_{0}, 0\right), 0\right)=\rho_{0}\left(x_{0}\right) \quad \text { for } \quad 0<\eta\left(t, x_{0}, t_{0}\right)<1 \quad \forall t \in[0, T] \\
& \rho(0, t)=\rho(\eta(t, 0, t), t)=\rho_{l}(t) \quad \text { for } \quad u(0, t)>0, \\
& \rho(1, t)=\rho(\eta(t, 1, t), t)=\rho_{r}(t) \quad \text { for } \quad u(1, t)<0 \text {. }
\end{aligned}
$$

The density does not increase along the characteristics, it decreases at most exponentially in the region with non-vanishing heat-source $q$. This implies the estimates $(2.3),(2.4),(2.5)$.

Now we state the main global existence result.

ThEOREM 2.1. Let (A1)-(A3) hold. Then for all $T>0$ there exists a unique solution $(\rho, v)-\rho$ piecewise continuous differentiable in $[0,1] \times[0, T]$ and $v \in C^{1}[0, T]-$ to the equations (1.10)-(1.11) with boundary conditions (1.6) and initial conditions $\left(\rho_{0}, v_{0}\right)$.

The proof of this theorem will be presented at the end of this section. As a preparation we present a lemma and the corresponding local in time existence result. The following lemma is an immediate consequence of lemma 2.1. It is stated without proof.

LEMMA 2.2. Under the above assumptions we have

$$
\begin{aligned}
0<\rho_{\min }(T) \leq & R(t), \\
& R_{q}(t) \leq \rho_{\max }(T) q_{\max }(T), \\
& R_{Q q}(t) \leq \rho_{\max }(T) q_{\max }^{2}(T), \\
& R_{Q_{t}}(t) \leq \rho_{\max }(T) q_{\max }(T) q_{t ; \max }(T) \quad \forall t \in[0, T] .
\end{aligned}
$$

In the following we omit the argument $T$ and $f_{\max }$ has to be understood as $f_{\max }=f_{\max }(T)$.

We start the existence analysis with a local (in time) result.

THEOREM 2.2. Let (A1)-(A3) hold. Then there exists a $\delta>0$ such that there is a unique solution $(\rho, v)-\rho$ piecewise continuous differentiable in $[0,1] \times[0, \delta]$ and $v \in C^{1}[0, \delta]$ - to the equations (1.10)-(1.11) with boundary conditions (1.6) and initial conditions $\left(\rho_{0}, v_{0}\right)$.

Proof. For the following analysis we consider the corresponding integrated equation (1.11)

$$
\begin{aligned}
v(t)= & v_{0}-\int_{0}^{t} \frac{1}{R(s)}\left\{R_{q}(s) v(s)\right. \\
& +\int_{0}^{1} p_{d v}(x) \rho(x, s) \frac{(v(s)+Q(x, s))|v(s)+Q(x, s)|}{2} d x \\
& \left.+R_{Q_{t}}(s)+R_{Q q}(s)+R_{f_{d} \sin \alpha}(s)-p_{l}(s)+p_{r}(s)\right\} d s .
\end{aligned}
$$


We use a standard fixed point procedure to show local (in time) existence and uniqueness of solutions of the equations (1.10)-(1.11). Let $\delta, \sigma>0$ and we consider the domain

$$
D=\left\{(v, t) \mid-\delta \leq t \leq \delta, \quad v_{0}-\sigma \leq v \leq v_{0}+\sigma\right\}
$$

and $v \in C\left(I_{\delta}\right)=C[-\delta, \delta]$. Then we choose a closed subset $Y \subset C\left(I_{\delta}\right)$ with

$$
Y=\left\{v \in C\left(I_{\delta}\right) \mid\left\|v-v_{0}\right\|_{C\left(I_{\delta}\right)} \leq \sigma\right\} .
$$

We define the operator

$$
S: Y \rightarrow C\left(I_{\delta}\right)
$$

with

$$
\begin{aligned}
(S v)(t)= & v_{0}-\int_{0}^{t} \frac{1}{R(s)}\left\{R_{q}(s) v(s)\right. \\
& +\int_{0}^{1} p_{d v}(x) \rho(x, s) \frac{(v(s)+Q(x, s))|v(s)+Q(x, s)|}{2} d x \\
& \left.+R_{Q_{t}}(s)+R_{Q q}(s)+R_{f_{d} \sin \alpha}(s)-p_{l}(s)+p_{r}(s)\right\} d s
\end{aligned}
$$

where $\rho$ is given by the solution of the continuity equation (1.10) with boundary and initial data satisfying (A2) and (A3).

The idea is to choose $\delta$ and $\sigma$ in such a way, that the mapping $S$ is a contraction.

We assume $\delta \leq T$. Using the definition of the operator $S$ we estimate

$$
\begin{aligned}
\left|(S v)(\delta)-v_{0}\right| \leq & \mid \int_{0}^{\delta} \frac{1}{R(s)}\left\{R_{q}(s) v(s)+\int_{0}^{1} p_{d v}(x) \rho(x, s) \frac{(v(s)+Q(x, s))|v(s)+Q(x, s)|}{2} d x\right. \\
& \left.+R_{Q_{t}}(s)+R_{Q q}(s)+R_{f_{d} \sin \alpha}(s)-p_{l}(s)+p_{r}(s)\right\} d s \mid \\
\leq & \frac{\delta}{\rho_{\min }}\left\{\rho_{\max } q_{\max }\left[v_{0}+\sigma\right]+\rho_{\max } p_{d v ; \max }\right. \\
& {\left[\sigma^{2}+2 \sigma\left(v_{0}+Q_{\max }\right)+\left(v_{0}+Q_{\max }\right)^{2}\right] } \\
& \left.+\rho_{\max } Q_{t ; \max }+\rho_{\max } Q_{\max } q_{\max }+\rho_{\max } f_{d}-\left(p_{l}-p_{r}\right)_{\max }\right\} .
\end{aligned}
$$

Given a $\sigma$ it is easy to choose a $\delta$ such that $\left\|S v-v_{0}\right\|_{C\left(I_{\delta}\right)} \leq \sigma$. Thus $S: Y \rightarrow Y$.

Next we show the contractivity of the mapping $S$. Clearly, the contractivity is used in the iterative scheme

$$
v^{i+1}=S v^{i}, \quad v^{0}=v_{0}, \quad i=0,1,2, \ldots,
$$

i.e. first we solve

$$
\begin{aligned}
& \frac{d \eta^{i}\left(t, x_{0}, t_{0}\right)}{d t}=v^{i}(t)+Q\left(\eta^{i}\left(t, x_{0}, t_{0}\right), t\right),\left\{\begin{array}{l}
\eta^{i}\left(0, x_{0}, 0\right)=x_{0} \\
\eta^{i}\left(t_{0}, 0, t_{0}\right)=0 \\
\eta^{i}\left(t_{0}, 1, t_{0}\right)=1
\end{array}\right. \\
& \frac{d \rho^{i}\left(\eta^{i}\left(t, x_{0}, t_{0}\right), t\right)}{d t}=-q\left(\eta^{i}\left(t, x_{0}, t_{0}\right), t\right) \rho^{i}\left(\eta^{i}\left(t, x_{0}, t_{0}\right), t\right), \\
& \rho^{i}\left(\eta^{i}\left(t_{0}, x_{0}, t_{0}\right), t_{0}\right)=\left\{\begin{array}{lll}
\rho_{l}\left(t_{0}\right) & \text { for } x_{0}=0 \\
\rho_{r}\left(t_{0}\right) & \text { for } & x_{0}=1 \\
\rho_{0}\left(x_{0}\right) & \text { for } & t_{0}=0
\end{array}\right.
\end{aligned}
$$


and then

$$
\begin{aligned}
v^{i+1}(\delta)= & v_{0}-\int_{0}^{\delta} \frac{1}{R^{i}(s)}\left\{R_{q}^{i}(s) v^{i}(s)\right. \\
& +\int_{0}^{1} p_{d v}(x) \rho^{i}(x, s) \frac{\left(v^{i}(s)+Q(x, s)\right)\left|v^{i}(s)+Q(x, s)\right|}{2} d x \\
& \left.+R_{Q_{t}}^{i}(s)+R_{Q q}^{i}(s)+R_{f_{d} \sin \alpha}^{i}(s)-p_{l}(s)+p_{r}(s)\right\} d s
\end{aligned}
$$

where we denote by

$$
R_{f}^{i}(\delta)=\int_{0}^{1} \rho^{i}(x, \delta) f(x, \delta) d x
$$

From now on we have to prove basically two things:

1. First we estimate possible changes in the density - or in the integrated density - due to the changes from iteration level $i-1$ to $i$ such that

$$
\int_{0}^{1}\left|\rho^{i}(x, t)-\rho^{i-1}(x, t)\right| d x \leq c t|| v^{i}-v^{i-1} \|_{C\left(I_{\delta}\right)}, \quad \forall t \in[0, \delta]
$$

where $c$ depends only on the data and

$$
\delta<\frac{1}{\max \left(\left|v_{0}+\sigma\right|,\left|v_{0}-\sigma\right|, q_{\max }\right)} .
$$

2. Then all terms in the iteration-expression $\left\|v^{i+1}-v^{i}\right\|_{C\left(I_{\delta}\right)}$ will be reduced to an estimate on the left hand side of (2.24).

We start with 1):

In a first step we assume that $\rho_{l}$ and $\rho_{r}$ are constant (in $t$ ). We choose a limit for the length of the time interval (2.24) and consider the interval $[0,1]$ at time $t \in[0, \delta]$.

In order to estimate the quantity

$$
\int_{0}^{1}\left|\rho^{i}(x, t)-\rho^{i-1}(x, t)\right| d x
$$

we split the the interval $[0,1]$ (at time $t$ ) in different regions. Let for $i=0,1,2, \ldots$

$$
\begin{aligned}
& x_{l}^{i}=\inf _{x_{0} \in[0,1]}\left\{\eta^{i}\left(t, x_{0}, 0\right) \mid 0<\eta^{i}\left(s, x_{0}, 0\right)<1 \forall s \in[0, t]\right\} \\
& x_{r}^{i}=\sup _{x_{0} \in[0,1]}\left\{\eta^{i}\left(t, x_{0}, 0\right) \mid 0<\eta^{i}\left(s, x_{0}, 0\right)<1 \forall s \in[0, t]\right\} .
\end{aligned}
$$

We denote by $x_{s}^{i}=\eta_{s}^{i}\left(t, x_{0 s}, 0\right), s=1,2, \ldots, S$ the value at $t$ of the characteristics which satisfies $x_{l}^{i} \leq \eta_{s}^{i}\left(t, x_{0 s}, 0\right) \leq x_{r}^{i}$, where $x_{0 s}, s=1,2, . . S$ are the discontinuities of the initial density $\rho_{0}$. We denote (see Figure 2.1)

$$
\begin{aligned}
\Omega_{b l} & =\left(0, \min \left(x_{l}^{i}, x_{l}^{i-1}\right)\right) \\
\Omega_{b r} & =\left(\max \left(x_{r}^{i}, x_{r}^{i-1}\right), 1\right) \\
\Omega_{l} & =\left(\min \left(x_{l}^{i}, x_{l}^{i-1}\right), \max \left(x_{l}^{i}, x_{l}^{i-1}\right)\right) \\
\Omega_{r} & =\left(\min \left(x_{r}^{i}, x_{r}^{i-1}\right), \max \left(x_{r}^{i}, x_{r}^{i-1}\right)\right) \\
\Omega_{d s} & =\left(\min \left(x_{s}^{i}, x_{s}^{i-1}\right), \max \left(x_{s}^{i}, x_{s}^{i-1}\right)\right), \quad s=1,2, \ldots, S . \\
\Omega_{c} & =(0,1)-\left\{\Omega_{b l} \cup \Omega_{b r} \cup \Omega_{l} \cup \Omega_{r} \bigcup_{s=1}^{S} \Omega_{d s}\right\} .
\end{aligned}
$$


To simplify the notation of the characteristic curves in the following we omit the arguments $x_{0}$ and $t_{0}$ and write simply $\eta^{i}(s)$ instead of $\eta^{i}\left(s, x_{0}, t_{0}\right)\left(x_{0}\right.$ and $t_{0}$ have to understood from the context). We estimate (due to (2.18))

$$
\max _{s \in\left[t_{0}, t\right]}\left|\eta^{i}(s)-\eta^{i-1}(s)\right| \leq \frac{1}{1-q_{\max }\left|t-t_{0}\right|}\left\{\left|t-t_{0}\right||| v^{i}-\left.v^{i-1}\right|_{C\left(I_{\delta}\right)}+\left|\eta^{i}\left(t_{0}\right)-\eta^{i-1}\left(t_{0}\right)\right|\right\}
$$

where the second estimate is to be intended along the characteristic $\eta^{i}$ or along the characteristic $\eta^{i-1}$. Thus for $\eta^{i}\left(t_{0}\right)=\eta^{i-1}\left(t_{0}\right)$ and under the condition (2.24)

$$
\max _{s \in[0, t]}\left|\eta^{i}(s)-\eta^{i-1}(s)\right| \leq\left|t-t_{0}\right||| v^{i}-v^{i-1}||_{C\left(I_{\delta}\right)}
$$

holds. It is easy to see that (constant boundary data for $\rho$ )

$$
\int_{\Omega_{b l}}\left|\rho^{i}(x, t)-\rho^{i-1}(x, t)\right| d x=\int_{\Omega_{b r}}\left|\rho^{i}(x, t)-\rho^{i-1}(x, t)\right| d x=0 .
$$

Applying (2.35) we obtain

$$
\begin{aligned}
\int_{\Omega_{d s}}\left|\rho^{i}(x, t)-\rho^{i-1}(x, t)\right| d x & \leq \rho_{\text {diff } ; \max } \max _{s \in[0, t]}\left|\eta_{s}^{i}(s)-\eta_{s}^{i-1}(s)\right| \\
& \leq \rho_{\text {diff } ; \max } t|| v^{i}-v^{i-1} \|_{C\left(I_{\delta}\right)}, \quad s=1,2, \ldots, S,
\end{aligned}
$$

where $\rho_{\text {diff;max }}=\rho_{\max }-\rho_{\min }$. Then we use

$$
\begin{aligned}
\frac{d}{d t}\left(\rho^{i}\left(\eta^{i}(t), t\right)-\rho^{i-1}\left(\eta^{i-1}(t), t\right)\right)= & -q\left(\eta^{i}(t), t\right) \rho^{i}\left(\eta^{i}(t), t\right)+q\left(\eta^{i-1}(t), t\right) \rho^{i-1}\left(\eta^{i-1}(t), t\right) \\
= & -q\left(\eta^{i-1}(t), t\right)\left[\rho^{i}\left(\eta^{i}(t), t\right)-\rho^{i-1}\left(\eta^{i-1}(t), t\right)\right] \\
& -\left[q\left(\eta^{i}(t), t\right)-q\left(\eta^{i-1}(t), t\right)\right] \rho^{i}\left(\eta^{i}(t), t\right) .
\end{aligned}
$$

Therefore (using $q \geq 0$ )

$$
\begin{aligned}
\left|\rho^{i}\left(\eta^{i}(t), t\right)-\rho^{i-1}\left(\eta^{i-1}(t), t\right)\right| \leq & \left|\rho^{i}\left(\eta^{i}(0), 0\right)-\rho^{i-1}\left(\eta^{i-1}(0), 0\right)\right| \\
& +q_{x ; \max } \rho_{\max } \max _{s \in[0, t]}\left|\eta^{i}(s)-\eta^{i-1}(s)\right| \\
\leq & t|| v^{i}-v^{i-1}||_{C\left(I_{\delta}\right)}\left(\max _{x \in[0,1]}\left|\left(\rho_{0}\right)_{x}(x)\right|+\left(q_{x}\right)_{\max } \rho_{\max }\right)
\end{aligned}
$$

and

$$
\int_{\Omega_{c}}\left|\rho^{i}(x, t)-\rho^{i-1}(x, t)\right| d x \leq t|| v^{i}-v^{i-1} \|_{C\left(I_{\delta}\right)}\left(\max _{x \in[0,1]}\left|\left(\rho_{0}\right)_{x}(x)\right|+\left(q_{x}\right)_{\max } \rho_{\max }\right) .
$$

It remains to estimate the differences on $\Omega_{l}$ and $\Omega_{r}$. We consider now $\Omega_{l}$ and assume that $x_{l}^{i}<x_{l}^{i-1}$. Then we consider the two characteristics $\eta_{l 1}^{i}$ and $\eta_{l 2}^{i}$ (at iteration level $i$ ) with $\eta_{l 1}^{i}(t)=x_{l}^{i}$ and $\eta_{l 2}^{i}(t)=x_{l}^{i-1}$. Due to (2.24) these two characteristics cannot reach the right boundary at $x=1$. There exists an $s \in[0, t]$ with $\eta_{l 1}^{i}(s)=0$ and $\left|\eta_{l 2}^{i}(s)\right| \leq|t|\left\|v^{i}-v^{i-1}\right\|_{C\left(I_{\delta}\right)}$ (same argument as in (2.35) for the characteristics $\eta_{l 2}^{i}$ and 


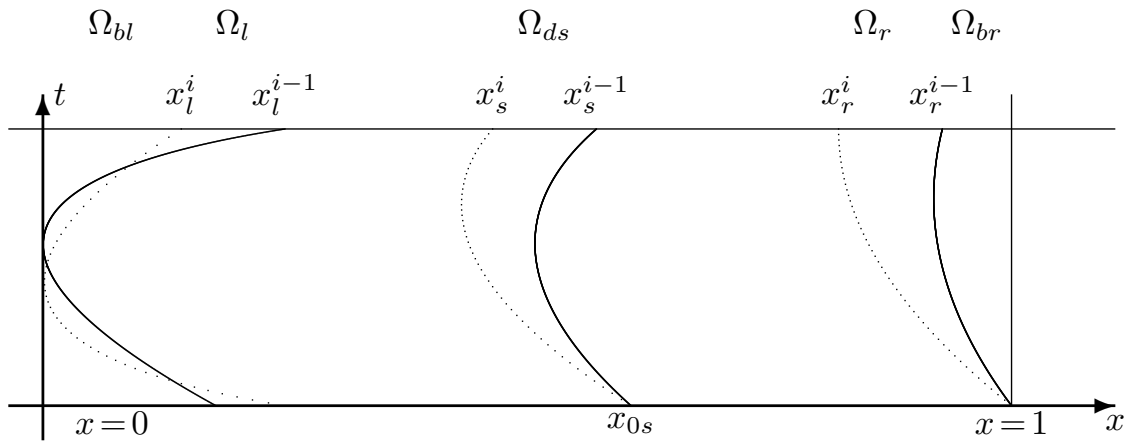

FIG. 2.1. Schematic view of the notations. The continuous and dashed lines correspond to the iteration level $i-1$ and $i$, respectively.

$\eta^{i-1}$ with $\left.\eta^{i-1}(t)=x_{l}^{i-1}\right)$. Since $\eta_{l 1}^{i}$ and $\eta_{l 2}^{i}$ do not cross we have $\left|\eta_{l 1}^{i}(s)-\eta_{l 2}^{i}(s)\right| \leq$ $|t||| v^{i}-v^{i-1} \|_{C\left(I_{\delta}\right)}$. Thus we conclude for the size of $\Omega_{l}$

$$
\left|x_{l}^{i}-x_{l}^{i-1}\right|=\left|\eta_{l 1}^{i}(t)-\eta_{l 2}^{i}(t)\right| \leq|t||| v^{i}-v^{i-1}||_{C\left(I_{\delta}\right)} e^{q_{\max } t}
$$

and therefore

$$
\int_{\Omega_{l}}\left|\rho^{i}(x, t)-\rho^{i-1}(x, t)\right| d x \leq \rho_{\text {diff; } \max }|t|\left\|v^{i}-v^{i-1}\right\|_{C\left(I_{\delta}\right)} e^{q_{\max } t} .
$$

The same estimate holds for $\Omega_{r}$. This leads to an estimate of the type $(t<\delta)$

$$
\int_{0}^{1}\left|\rho^{i}(x, t)-\rho^{i-1}(x, t)\right| d x \leq c t\left\|v^{i}-v^{i-1}\right\|_{C\left(I_{\delta}\right)},
$$

where $c$ depends on $T$ and on the data. Now we turn to the case of non-constant boundary data. In this case we transform the problem by using

$$
\bar{\rho}(x, t)=\rho(x, t)-\left[(1-x) \rho_{l}(t)+x \rho_{r}(t)\right]
$$

into

$$
\bar{\rho}_{t}^{i}+u^{i} \bar{\rho}_{x}^{i}=-q \bar{\rho}^{i}-u^{i}\left(\rho_{r}-\rho_{l}\right)-\left[(1-x)\left(\rho_{l}\right)_{t}+x\left(\rho_{r}\right)_{t}\right]-q\left[(1-x) \rho_{l}+x \rho_{r}\right]
$$

with constant boundary data

$$
\bar{\rho}(0, t)=0, \quad \bar{\rho}(1, t)=0, \quad \bar{\rho}(x, 0)=\rho_{0}(x)-\left[(1-x) \rho_{l}(0)+x \rho_{r}(0)\right] .
$$

Now we use the same estimation procedure as above in the case of constant boundary data. The difference lies in the fact that the right hand side in the equation (2.45) has changed. We see in (2.18) that the characteristic curves remain unchanged. The only changes appear in (2.39)-(2.42) where the densities are involved. Equation (2.39) remains unchanged due to

$$
\bar{\rho}^{i}(x, t)-\bar{\rho}^{i-1}(x, t)=\rho^{i}(x, t)-\rho^{i-1}(x, t) .
$$


In (2.41) and (2.42) the quantity $\rho_{\text {dif } f ; \max }$ has to be revisited. Also $\rho_{\max }$ in $(2.41)$ and $(2.42)$ has to be replaced by the corresponding value of $\bar{\rho}$. It is easy to see that

$$
\begin{aligned}
\left|\bar{\rho}^{i}(x, t)\right| \leq \bar{\rho}_{\max }= & \bar{\rho}_{0 ; \max }+t\left[\left(\rho_{l}-\rho_{r}\right)_{\max }\left(\left|v_{0}+\sigma\right|+q_{\max }\right)\right. \\
& +q_{\max }\left(\rho_{l ; \max }+\rho_{r ; \max }+\left(\rho_{l t ; \max }+\rho_{r t ; \max }\right]\right.
\end{aligned}
$$

and therefore $\bar{\rho}_{\text {diffmax }} \leq 2 \bar{\rho}_{\max }$. Along these lines we obtain instead of (2.39)

$$
\begin{aligned}
\left|\bar{\rho}^{i}\left(\eta^{i}(t), t\right)-\bar{\rho}^{i-1}\left(\eta^{i-1}(t), t\right)\right| \leq & t\left\|v^{i}-v^{i-1}\right\|_{C\left(I_{\delta}\right)}\left[\bar{\rho}_{0 x ; \max }+q_{x ; \max } \bar{\rho}_{\max } t\right. \\
& +\left(\rho_{l}-\rho_{r}\right)_{\max }\left(1+q_{\max } t+q_{\max }\left(\rho_{l ; \max }+\rho_{r ; \max }\right.\right. \\
& +\left(\rho_{l t ; \max }+\rho_{r t ; \max }\right] .
\end{aligned}
$$

Putting all the estimates together we finally obtain the desired result

$$
\int_{0}^{1}\left|\rho^{i}(x, t)-\rho^{i-1}(x, t)\right| d x \leq c t|| v^{i}-v^{i-1} \|_{C\left(I_{\delta}\right)}, \quad \forall t \leq \delta
$$

where $c$ depends only on the data.

Now we pass to 2):

In the following all terms to be estimated in the iteration-expression $\left\|v^{i+1}-v^{i}\right\|_{C\left(I_{\delta}\right)}$ will be reduced to an estimate on the left hand side of (2.23). We start with

$$
\int_{0}^{\delta}\left|R_{f}^{i}(s)-R_{f}^{i-1}(s)\right| d s \leq \delta f_{\max } \max _{s \in[0, \delta]} \int_{0}^{1}\left|\rho^{i}(x, s)-\rho^{i-1}(x, s)\right| d x
$$

and

$$
\begin{aligned}
\int_{0}^{\delta}\left|\frac{R_{f}^{i}(s)}{R^{i}(s)}-\frac{R_{f}^{i-1}(s)}{R^{i-1}(s)}\right| d s \leq & \int_{0}^{\delta}\left|\frac{R_{f}^{i}(s) R^{i-1}(s)-R^{i}(s) R_{f}^{i-1}(s)}{R^{i}(s) R^{i-1}(s)}\right| d s \\
\leq & \frac{\rho_{\max }}{\rho_{\min }^{2}} \int_{0}^{\delta}\left|R_{f}^{i}(s)-R_{f}^{i-1}(s)\right| d s \\
& +\frac{\rho_{\max } f_{\max }}{\rho_{\min }^{2}} \int_{0}^{\delta}\left|R^{i}(s)-R^{i-1}(s)\right| d s
\end{aligned}
$$

and

$$
\begin{aligned}
\left|\int_{0}^{\delta}\left(\frac{R_{q}^{i}(s)}{R^{i}(s)} v^{i}(s)-\frac{R_{q}^{i-1}(s)}{R^{i-1}(s)} v^{i-1}(s)\right) d s\right| \leq & \int_{0}^{\delta} \frac{R_{q}^{i}(s)}{R^{i}(s)}\left|v^{i}(s)-v^{i-1}(s)\right| d s \\
& +\int_{0}^{\delta}\left|v^{i-1}(s)\right|\left|\frac{R_{q}^{i}(s)}{R^{i}(s)}-\frac{R_{q}^{i-1}(s)}{R^{i-1}(s)}\right| d s \\
\leq & \delta \frac{\rho_{\max } q_{\max }}{\rho_{\min }}\left\|v^{i}-v^{i-1}\right\|_{C\left(I_{\delta}\right)} \\
& +\left[v_{0}+\sigma\right] \int_{0}^{\delta}\left|\left(\frac{R_{q}^{i}(s)}{R^{i}(s)}-\frac{R_{q}^{i-1}(s)}{R^{i-1}(s)}\right)\right| d s .
\end{aligned}
$$


Analogously

$$
\begin{aligned}
& \mid \int_{0}^{\delta} \int_{0}^{1} p_{d v}(x)\left(\rho^{i}(x, s) \frac{\left.\left(v^{i}(s)+Q(x, s)\right) \mid v^{i}(s)+Q(x, s)\right) \mid}{R^{i}(s)}\right. \\
& \left.\quad-\rho^{i-1}(x, s) \frac{\left.\left(v^{i-1}(s)+Q(x, s)\right) \mid v^{i-1}(s)+Q(x, s)\right) \mid}{R^{i-1}(s)}\right) d x d s \mid \\
& \leq \int_{0}^{\delta} \int_{0}^{1} p_{d v}(x) \frac{1}{R^{i}(s)}\left|\rho^{i}(x, s)-\rho^{i-1}(x, s)\right|\left(v^{i}+Q(x, s)\right)^{2} d x d s \\
& +\int_{0}^{\delta} \int_{0}^{1} p_{d v}(x) \frac{\rho^{i-1}(x, s)}{R^{i}(s)}\left|v^{i}(x, s)-v^{i-1}(x, s)\right|\left|v^{i}+Q(x, s)\right| d x d s \\
& +\int_{0}^{\delta} \int_{0}^{1} p_{d v}(x) \frac{\rho^{i-1}(x, s)}{R^{i}(s)}\left|\left(v^{i}+Q(x, s)\right)\left(\left|v^{i}(x, s)+Q(x, s)\right|-\left|v^{i-1}(x, s)-Q(x, s)\right|\right)\right| d x d s \\
& +\int_{0}^{\delta} \int_{0}^{1} p_{d v}(x)\left|\frac{1}{R^{i}(s)}-\frac{1}{R^{i-1}(s)}\right| \rho^{i-1}(x, s)\left(v^{i}(s)+Q(x, s)\right)^{2} d x d s \\
& \leq p_{d v ; \max } \frac{1}{\rho_{\min }}\left(v_{0}+\sigma+q_{\max }\right)^{2} \int_{0}^{\delta} \int_{0}^{1}\left|\rho^{i}(x, s)-\rho^{i-1}(x, s)\right| d x d s \\
& +2 p_{d v ; \max } \frac{\rho_{\max }}{\rho_{\min }}\left(v_{0}+\sigma+q_{\max }\right) \delta \| v^{i}-v_{i-1}|| C\left(I_{\delta}\right) \\
& \quad+p_{d v ; \max } \rho_{\max }\left(v_{0}+\sigma+q_{\max }\right)^{2} \int_{0}^{\delta} \int_{0}^{1}\left|\frac{1}{R^{i}(s)}-\frac{1}{R^{i-1}(s)}\right| d x d s .
\end{aligned}
$$

Summarizing the estimates (2.23)-(2.54) we obtain

$$
\left\|v^{i+1}-v^{i}\right\|_{C\left(I_{\delta}\right)} \leq c \delta\left\|v^{i}-v^{i-1}\right\|_{C\left(I_{\delta}\right)}, \quad i=1,2, \ldots,
$$

where the constant $c$ depends only on the data, on $\delta$ and on $T$. Therefore, given a $\sigma$ choosing $\delta$ small enough the map $S$ turns out to be a contraction.

The properties a) and b) allow us to conclude that there is a unique fixed point of the map $S$ in $Y$ which represents the solution. This concludes the proof.

At the end we conclude with the proof of the main Theorem 2.1:

Proof. Here we have to show that the solution is extendible to the interval $t \in[0, T]$. We multiply (1.11) by $\frac{v}{|v|}$ and obtain (for $v \neq 0$ )

$$
|v|_{t}+\frac{R_{q}}{R}|v|=-\int_{0}^{1} p_{d v} \rho \frac{v}{|v|} \frac{(v+Q)|v+Q|}{2} d x-\frac{v}{|v|} \frac{1}{R}\left\{R_{Q_{t}}+R_{Q q} R_{f_{d} \sin \alpha}-p_{l}+p_{r}\right\} .
$$

Obviously, the last term on the right hand side is bounded. The first term on the right hand side can be estimated

$$
-\int_{0}^{1} p_{d v} \rho \frac{v}{|v|} \frac{(v+Q)|v+Q|}{2} d x \leq\left\{\begin{array}{clc}
0 & \text { for } & v \geq 0 \\
p_{d v ; \max } \rho_{\max } q_{\max }^{2} & \text { for } & -q_{\max }<v<0 \\
0 & \text { for } & v \leq-q_{\max }
\end{array}\right.
$$

Therefore, $|v|$ grows at most exponentially and exists for every finite time interval. Since also $q \geq 0$ holds, $|v|$ remains bounded. This concludes the proof. 


\section{Conclusions}

We have shown a global existence and uniqueness result for solutions of model equations for the description of tunnel fires. This result is a necessary basis for ongoing studies on the model. A possible extension is to study tunnel-networks. In this case we need a good knowledge of the model for the single "network-pieces". Another extension is to introduce control-mechanisms and to study the resulting dynamics.

Acknowledgement. The first author was partially supported by the Berufsfeuerwehr Bozen and by the EU network Hyperbolic and Kinetic Equations HYKE (Contract number: HPRN-CT-2002-00282).

\section{REFERENCES}

[1] http://www.etnfit.net/ (6th September 2004).

[2] I. Gasser, An asymptotic-induced one-dimensional model to describe fires in tunnels II: the stationary model, Math. Meth. in the Appl. Sci. $M^{2}$ AS, 26(15), 1327-1347, 2003.

[3] I. Gasser, On the mathematics of tunnel fires, Mitteilungen der GAMM., 26(1-2), 109-126, 2003.

[4] G. B. Brandt, S. F. Jagger and C. J. Lea, Fires in tunnels, Phil. Trans. R. Soc. Lond. A, 356, 2873-2906, 1998

[5] I. Gasser and H. Steinrück, An asymptotic-induced one-dimensional model to describe fires in tunnels III: the transient problem, SIAM J. Appl. Math., to appear, 2006.

[6] I. Gasser and J. Struckmeier, An asymptotic-induced one-dimensional model to describe fires in tunnels, Math. Meth. in the Appl. Sci. $M^{2} A S, 25(14), 1231-1249,2002$.

[7] S. M. Olenick and D. J. Carpenter, An updated international survey of computer models for fire and smoke, J. of Fire Prot. Engr., 13(2), 87-110, 2003.

[8] PIARC 1999 Fire and Smoke Control in Road Tunnels, PIARC Commettee on road tunnels (C 5), 05.05.B, World Road Association, 1999. 